\title{
Distribution of current density, temperature and mechanical deformation in YBCO bulks under Field-Cooling magnetization
}

\author{
Frederic Trillaud, Kévin Berger, Bruno Douine and Jean Lévêque
}

\begin{abstract}
Most of the latest models of HTS bulk magnetization take into account the thermal and electromagnetic coupling, but, they still lack the implementation of the mechanical deformation under strong magnetic fields. The following work tries to fill up partly the gap by proposing a model of the dependence of the electrical conductivity upon mechanical deformation during Field-Cooling magnetization. This dependence is carried out by the critical current density as a function of induced thermal and mechanical strain in the bulk. As a first approximation and bearing in mind the obvious discrepancies, a simple model inferred from HTS thin films and generic knowledge on ceramics is proposed. This first approach allows drawing some generalities on the expected thermal, magnetic and mechanical response of disk-shaped YBCO pellets during magnetization. The utter goal is to assess design parameters to insert those pellets in electrical rotatory machines.
\end{abstract}

Index Terms-magnetization, mechanical analysis, YBCO bulk modelling.

\section{INTRODUCTION}

$\mathbf{T}$ HE magnetization of HTS pellets is a delicate process that involves cooling a single grown bulky ceramic compound from room temperature to cryogenic temperatures and sweeping it with an external magnetic field to generate large induced currents. Ceramics being brittle materials, the first procedure is often the most critical one. Indeed, thermal induced mechanical deformations lead to the formation and/or the propagation of new and/or pre-exiting cracks that ultimately limit the capability of the bulk to generate the required level of induced current to trap most of the applied external field [1], [2]. Then, during the magnetization, the combination of a large current density of the order of the critical current density and a large resulting magnetic flux density in the bulk induces local mechanical stresses which may propagate farther the cracks, degrading further the magnetic trapping [3]. The phenomena involved are fairly non-linear leading to a large increase in complexity of the multi-physics problem. The present work proposes a model simple enough to capture some first insights on the impact of mechanical deformation

Manuscript submitted on September 19, 2017. This work was supported in part by DGAPA-UNAM grant, PAPIIT-2017 No. TA100617. (corresponding author: F. Trillaud).

F. Trillaud is with the Institute of Engineering, National Autonomous University of Mexico, CDMX, 04510 Mexico (e-mail: ftrillaudp@ii.unam.mx).

K. Berger, B. Douine and J. Lévêque are with the University of Lorraine, Groupe de Recherche en Energie Electrique de Nancy, 54506, Vandoeuvrelès-Nancy, France.

Color versions of one or more of the figures in this paper are available online at http://ieeexplore.ieee.org. on the distribution of current density and subsequently on the magnitude of trapped magnetic field without having to detail the generation and the propagation of cracks.

The proposed model describes the coupled electromagnetic, thermal and mechanical behaviour of a homogeneous YBCO pellet. The electromagnetic model which relies on the Aformulation of the Maxwell equations was presented in [4]. This model was modified to include both the dependence of the critical current density upon mechanical strain and temperature, in addition to the original dependence upon magnetic flux density, and the dependence of the $n$-value upon temperature and magnetic flux density as well. The latter improvement allowed exploring the behavior of the pellet under large magnetic flux densities where the $n$-value decreases to the point that the assumptions of the critical state model do not hold anymore (typically, $n<15$ ). An ideal mechanical model of the bulk was inferred from generic knowledge on the stress-strain relation of ceramics [5], [6] and the strain dependence of superconducting wires and tapes [7], [8]. In this model, the bulk shows a linear elastic behaviour until failure occurs at a characteristic fracture strength of the material. The mechanical strain and the resulting stress are obtained from the Von Mises' theory [9].

\section{MOdELING OF THE PROBLEM}

\section{A. Geometry and assumptions}

Fig. 1 shows the schematic dimensional drawing of the YBCO pellet and its surrounding. This figure corresponds to the actual setup that was used to carried out the experimental work. Not shown in the illustration, a Hall sensor was located at $1.5 \mathrm{~mm}$ above the top, center surface of the bulk and a thermal sensor at the bottom of the sample casing.

To simplify the problem, several basic assumptions were considered. The bulk is assumed to be homogeneous having orthotropic material properties. The mechanical behaviour of the bulk can be entirely described by a functional dependence of the critical current density $J_{\mathrm{c}}$ upon the equivalent strain $\epsilon_{\text {eq }}$, the local temperature $T$ and the resulting magnetic flux density $B_{\mathrm{r}}$. Therefore, the model, which includes the bulk, the surrounding vacuum and the background electromagnet, was reduced to only one half of the axisymmetric initial one.

The geometry and its mesh were built using the 3D modeller Gmsh [10]. The numerical coupled axisymmetric problem was solved by the Finite Element Method (FEM) solver GetDP [11]. 


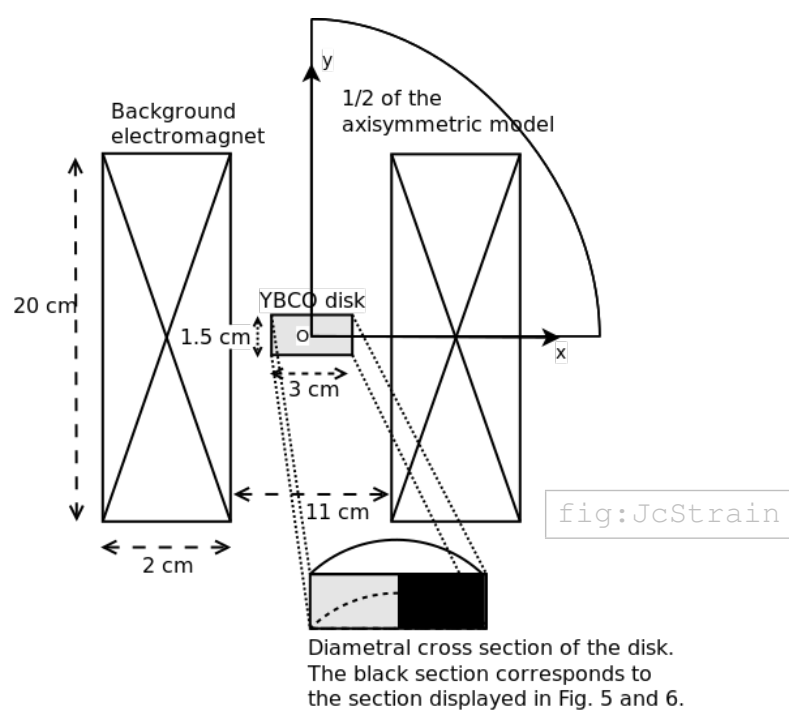

Fig. 1. Partial illustration of the experimental setup and its corresponding model. In the actual experiment, the sample is surrounded by a brass casing connected to a single-stage cryocooler to provide temperature homogenization and mechanical support.

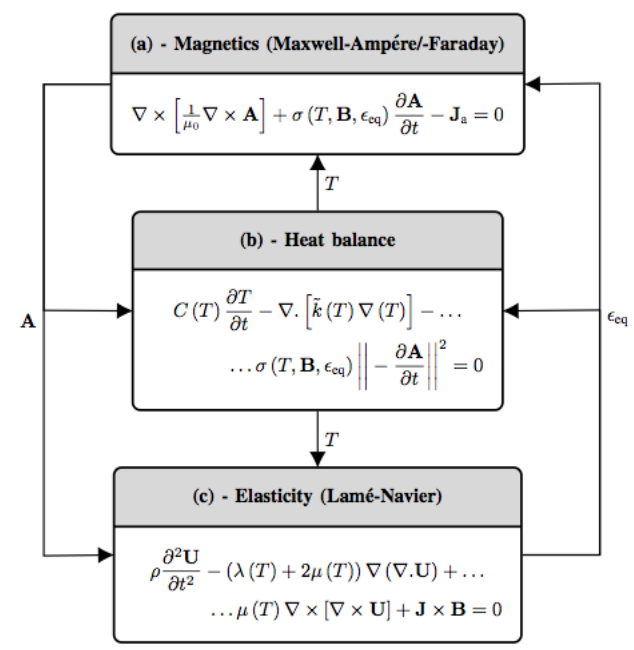

Fig. 2. Layout of the coupling between the electromagnetic (a), thermal (b) and mechanical (c) equations. The coupling is carried by the magnetic vector potential $\mathbf{A}$, the temperature $T$ and the equivalent strain $\epsilon_{e q}$. The magnetic flux density and the induced current are given by $\mathbf{B}=\nabla \times \mathbf{A}$ and $\mathbf{J}=-\sigma \partial \mathbf{A} / \partial t$, respectively. $\rho$ and $\mathbf{J}_{\mathrm{a}}$ are the mass density and the electromagnet current density, respectively.

\section{B. Coupled thermal, mechanical and electromagnetic model}

Fig. 2 provides the coupling between the different equations involved in the multiphysics problem. A brief summary of the electromagnetic and thermal models and a more detailed description of the mechanical one are provided in the next paragraphs.

a) Electromagnetic model: A detailed description of the electromagnetic model is given in [4]. This model expresses the Maxwell equations using the magnetic vector potential $\mathbf{A}$. The initial model was modified to include the dependence of the electrical conductivity $\sigma$ of the superconductor on temperature and equivalent strain in addition to the magnetic flux density.

The general expression of the electrical conductivity is given

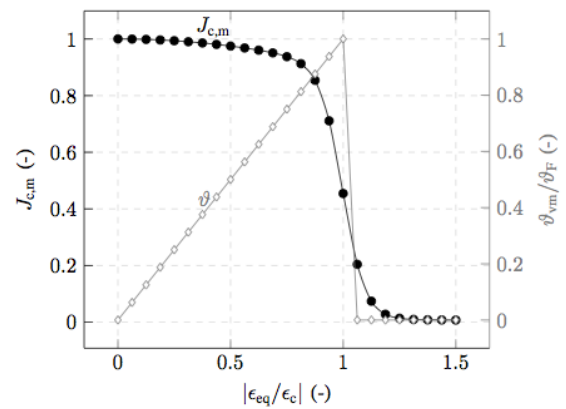

Fig. 3. $J_{\mathrm{c}, \mathrm{m}}$ versus the absolute value of $\epsilon_{\mathrm{eq}}$ normalized to $\epsilon_{\mathrm{c}}$. The ideal normalized stress-strain is also provided.

by

$$
\sigma=J_{\mathrm{c}} E_{\mathrm{c}}^{-\frac{1}{n}}\left(\left\|\frac{\partial \mathbf{A}}{\partial t}\right\|+10^{-6} E_{\mathrm{c}}\right)^{\frac{1-n}{n}}
$$

where the critical electric field, $E_{\mathrm{c}}$, is chosen equal to $1 \mu \mathrm{V} / \mathrm{cm}$. The critical current density $J_{\mathrm{c}}$ is divided in three terms as follows

$$
J_{\mathrm{c}}\left(T, B, \epsilon_{\mathrm{eq}}\right)=J_{\mathrm{c}, 0} \times J_{\mathrm{c}, \mathrm{TB}} \times J_{\mathrm{c}, \mathrm{m}}
$$

where $J_{\mathrm{c}, 0}\left(T_{\mathrm{ref}}, S F, \epsilon_{\mathrm{eq}}=0\right.$ ) (equal to $11 \mathrm{kA} / \mathrm{cm}^{2}$ ) is the self-field, strain-free critical current density at the reference temperature $T_{\text {ref }}=77 \mathrm{~K} . J_{\mathrm{c}, \text { ТB }}(T, B)$ is the temperature and magnetic flux density dependent strain-free critical current density given in the shape of a modified Kim-Anderson relation [4], [12], and $J_{\mathrm{c}, \mathrm{m}}\left(\epsilon_{\mathrm{eq}}\right)$ is the strain-dependent critical current density. Their respective expressions as well as the definition of the $n$-value are given below,

$$
J_{\mathrm{c}, \text { ТВ }}=\left(\frac{T-T_{\mathrm{c}}}{T_{\text {ref }}-T_{\mathrm{c}}}\right)^{0.96} \times\left(1+\frac{B}{B_{0}(T)}\right)^{-0.7}
$$

with,

$$
B_{0}=1.73 \times\left[1-\left(\frac{T}{T_{\mathrm{c} 0}}\right)^{2.17}\right]
$$

The different values appearing in the above equations were obtained by using available plot of normalized critical current versus background magnetic field and by fitting the experimental data of the field-cooling magnetization over time at various operating temperatures, as described in [4]; and

$$
J_{\mathrm{c}, \mathrm{m}}=\left[1-\chi\left(\frac{\epsilon_{\mathrm{eq}}}{\epsilon_{\mathrm{c}}}\right)^{2}\right] \times\left[\alpha+\left(\frac{1-\alpha}{1+\exp \left(\frac{\left|\frac{\epsilon_{\mathrm{eq}}}{\epsilon_{\mathrm{c}}}\right|-1}{\beta}\right)}\right)\right]
$$

with $\chi=0.1, \beta=0.025$ and $\alpha=10 \%$. Fig. 3 shows the resulting dependence of $J_{\mathrm{c}, \mathrm{m}}$ as a function of the normalized equivalent strain. The critical strain $\epsilon_{\mathrm{c}}$ is defined by the fracture strength $\vartheta_{\mathrm{F}}$ as $\epsilon_{\mathrm{c}}=\vartheta_{\mathrm{F}} / E$, where $E$ is the Young's modulus of the bulk. For YBCO bulks, at $77 \mathrm{~K}$, the fracture strength is of the order of $90 \mathrm{MPa}[13]$ and the Young's modulus is about $150 \mathrm{GPa}$ [14]. The model tries to catch up the actual behaviour of the sample by showing a degradation before reaching rupture. To that end, the $J_{\mathrm{c}, \mathrm{m}}$ starts degrading at 
about $90 \%$ of the fracture strength which can be considered as an optimistic value compared to the reported ultimate tensile strength of the order of 10 to $20 \mathrm{MPa}$ [6].

The $n$-value does not dependent upon the strain in the model. However, a temperature and magnetic flux density dependence is taken into account as proposed in [15],

$$
n=\left(n_{1}+\frac{n_{0}-n_{1}}{1+\frac{B}{B_{00}}}\right) \times\left(\frac{2}{1+\frac{T}{T_{\text {ref }}}}\right)
$$

with $n_{0}=30$ (at $\left.77 \mathrm{~K}, 0 \mathrm{~T}\right), n_{1}=5$ (at $77 \mathrm{~K}$, large magnetic fields), $B_{00}=0.5 \mathrm{~T}$ (at $n=\left(n_{0}+n_{1}\right) / 2$ ). These values were chosen to reproduce the trend of the $n$-value as a function of magnetic flux density and temperature $[16]-[18]$.

b) Thermal model: The thermal model which is coupled to the electromagnetic model through the dissipating term $Q=\sigma\|\partial \mathbf{A} / \partial t\|^{2}$ includes temperature-dependent material properties. The volumetric heat capacity $C$ and the diagonal tensor of thermal conductivity

$$
\tilde{k}=\left(\begin{array}{cc}
k_{\mathrm{ab}} & 0 \\
0 & k_{\mathrm{c}}
\end{array}\right)
$$

were fitted from averaged results found in $[19 !-22]$. Two thermal boundaries were considered, the thermal conduction through the brass casing connected to the single-stage cryocooler and adiabatic conditions. The former was used to reproduce the experimental data whereas the latter was applied to extrapolate the behaviour of the bulk to greater applied magnetic fields.

c) Thermoelastic model: The mechanical model is coupled to the electromagnetic model via the Lorentz force $\mathbf{J} \times \mathbf{B}$, and the thermal induced stress is added via the hooke's law. The space distribution and time evolution of the deformation vector $\mathbf{U}(x, y, t)=\left\{u_{\mathrm{x}}, u_{\mathrm{y}}, u_{\mathrm{xy}}\right\}$ is related to the stress vector $\vartheta=\left\{\vartheta_{\mathrm{x}}, \vartheta_{\mathrm{y}}, \vartheta_{\mathrm{xy}}\right\}$ by the following linear equation

$$
\boldsymbol{\vartheta}=\tilde{E}\left(\boldsymbol{\epsilon}_{\mathrm{m}}-\boldsymbol{\epsilon}_{\mathrm{th}}\right)
$$

where $\boldsymbol{\epsilon}_{\mathrm{m}}=\mathcal{D}(\mathbf{U})$ is the mechanical strain, and $\boldsymbol{\epsilon}_{\mathrm{th}}=$ $\left\{\alpha_{\mathrm{ab}} \Delta T, \alpha_{\mathrm{c}} \Delta T, 0\right\}$ is the thermal induced strain for a local increase in temperature, $\Delta T=T-T_{\text {ref }}[23] . \alpha_{\mathrm{ab}}$ and $\alpha_{\mathrm{c}}$ are the directional linear thermal expansion coefficients of the bulk in $\mathrm{K}^{-1}[24$. $\mathcal{D}$ is the symmetric gradient which is expressed as

$$
\mathcal{D}=\left(\begin{array}{ccc}
\frac{\partial}{\partial x} & 0 & \frac{\partial .}{\partial x} \\
0 & \frac{\partial .}{\partial y} & \frac{\partial \cdot}{\partial y} \\
0 & 0 & 0
\end{array}\right)^{T}
$$

$\tilde{E}$ is a symmetric tensor lumping the mechanical properties of the sample whose expression is given by

$$
\tilde{E}=\left(\begin{array}{ccc}
\lambda+2 \mu & \lambda & 0 \\
\lambda & \lambda+2 \mu & 0 \\
0 & 0 & \mu
\end{array}\right)
$$

The Lamé's coefficients $\lambda$ and $\mu$ are $\frac{\nu E}{(1-\nu)(1+2 \nu)}$ and $\frac{\nu E}{(1+2 \nu)}$, respectively [25]. In this representation, $\mu$ corresponds

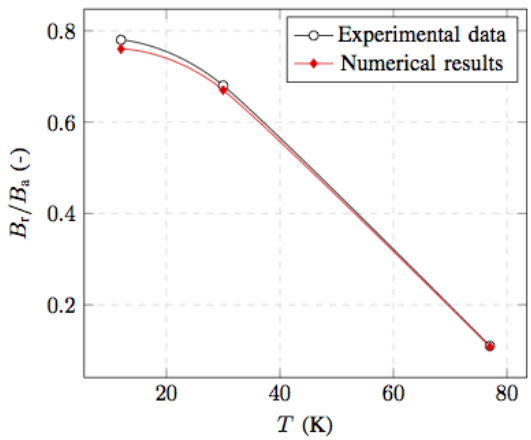

Fig. 4. Comparison of experimental data and numerical results (see Table II. $\dot{B}_{\mathrm{r}}$ and $B_{\mathrm{a}}$ are the resulting and applied magnetic flux densities, respectively.

TABLE I. Comparison between experimental data (ex) and numerical results $(\mathrm{nu})$ at a ramp rate of $0.003 \mathrm{~T} / \mathrm{s}$, in conduction cooling.

\begin{tabular}{|c|c|c|c|c|c|c|}
\hline \hline $\begin{array}{c}T_{\mathrm{op}} \\
(\mathrm{K})\end{array}$ & $\begin{array}{c}B_{\mathrm{a}} \\
(\mathrm{T})\end{array}$ & $\begin{array}{c}B_{\mathrm{r}}^{e x} / B_{\mathrm{a}} \\
(\%)\end{array}$ & $\begin{array}{c}B_{\mathrm{r}}^{n u} / B_{\mathrm{a}} \\
(\%)\end{array}$ & $\begin{array}{c}J_{\max }^{n u} / J_{\mathrm{c}} \\
(\%)\end{array}$ & $\begin{array}{c}\Delta T_{\max }^{n u} \\
(\mathrm{~K})\end{array}$ & $\begin{array}{c}\vartheta_{\mathrm{vm}, n u}^{\mathrm{av}} \\
(\mathrm{MPa})\end{array}$ \\
\hline 12 & 3.4 & 78 & 76 & 96.2 & 9.3 & 10 \\
\hline 30 & 3.08 & 68 & 67 & 95.8 & 2.5 & 7 \\
\hline 77 & 3.4 & 10.8 & 11 & 94 & $\tilde{0}$ & 0.2 \\
\hline \hline
\end{tabular}

TABLE II. Numerical results obtained for $T_{\mathrm{op}}=30 \mathrm{~K}$ at a ramp rate of $0.003 \mathrm{~T} / \mathrm{s}$ for $\vartheta_{\mathrm{F}}=90 \mathrm{MPa}$, in adiabatic conditions.

\begin{tabular}{|c|c|c|c|c|}
\hline \hline$B_{\mathrm{a}}(\mathrm{T})$ & $B_{\mathrm{t}} / B_{\mathrm{a}}(\%)$ & $J_{\max } / J_{\mathrm{c}}(\%)$ & $\Delta T_{\max }(\mathrm{K})$ & $\vartheta_{\mathrm{vm}}^{\mathrm{av}} / \vartheta_{\mathrm{F}}(\%)$ \\
\hline 3.08 & 67 & 95.8 & 2.5 & 11 \\
\hline 10 & 20 & 95.6 & 5.4 & 18 \\
\hline 15 & 13 & 114 & 6.4 & 22 \\
\hline \hline
\end{tabular}

to the shear modulus $G, E$ is the Young's modulus and $\nu$ is the Poisson's ratio equal to 0.255 [26]. The azimuthal component of the strain, $u_{\mathrm{x}} / x$, was computed at the post-processing stage.

\section{RESULTS AND DISCUSSION}

The proposed model was successfully cross-checked with available experimental data obtained during Field-Cooling magnetization at a ramp rate of $0.003 \mathrm{~T} / \mathrm{s}$. For this verification, the brass casing and its connection to the cold head was modelled. Fig. 4 in conjunction with Table II provides a comparison between the experimental and numerical results. A good matching was achieved. For this case study, due to the low magnitude of background magnetic flux densities $B_{\mathrm{a}}$, the incidence of the mechanical deformation on the results was found negligible. Starting from the experimental result obtained at $30 \mathrm{~K}$ and considering adiabatic conditions, greater $B_{\mathrm{a}}$ were then explored. Table [II summarizes some of the results for different $B_{\mathrm{a}}$ and a fracture strength $\vartheta_{\mathrm{F}}$ equal to $90 \mathrm{MPa}$. In that particular case, the average stress $\vartheta_{\mathrm{vm}}^{\mathrm{av}}$ remained well below the fracture strength. Therefore, the magnitude of the trapped magnetic flux density was solely determined by the heat dissipation generated in the bulk. Consequently, as the background field was increased, the electrical conductivity $\sigma$ and the fraction of total magnetic flux density over background magnetic flux density $B_{\mathrm{r}} / B_{\mathrm{a}}$ lowered. Fig. 5 shows the axisymmetric distributions of the normalized critical current density $J / J_{\mathrm{c}}$, the temperature $T$ and the deformation 


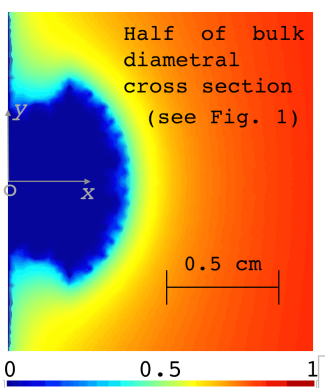

(a) $J / J_{\mathrm{c}}(-)$ at $t_{\mathrm{i}}$

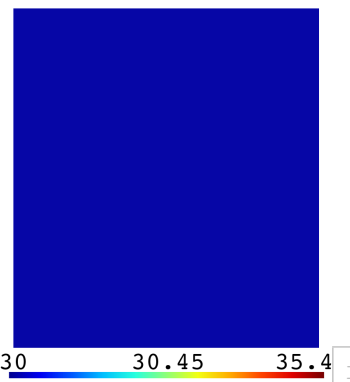

(c) $T(\mathrm{~K})$ at $t_{\mathrm{i}}$

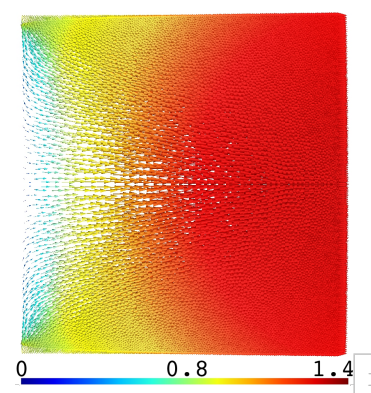

(e) $\mathbf{U}(\mu \mathrm{m})$ at $t_{\mathrm{i}}$

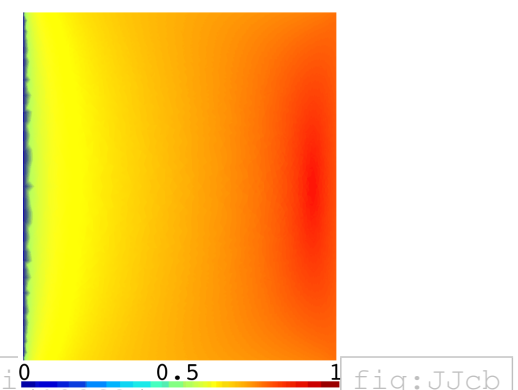

(b) $J / J_{\mathrm{c}}(-)$ at $t_{\mathrm{f}}$

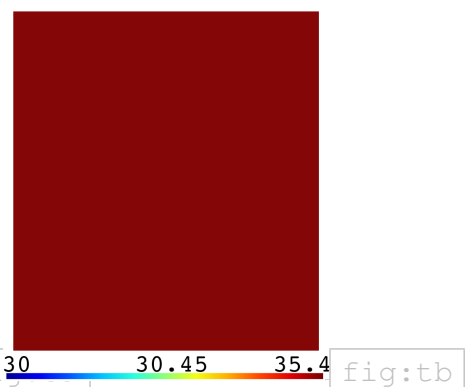

(d) $T(\mathrm{~K})$ at $t_{\mathrm{f}}$

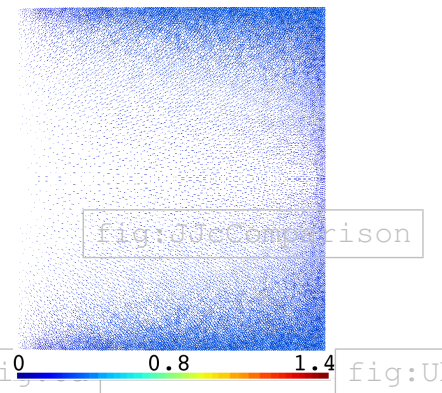

(f) $\mathbf{U}(\mu \mathrm{m})$ at $t_{\mathrm{f}}$

Fig. 5. Axisymmetric distribution of $J / J_{\mathrm{c}}(-), T$ and $\mathbf{U}$ (in $\mu \mathrm{m}$ ) in the bulk, for $T_{\mathrm{op}}=30 \mathrm{~K}, B_{\mathrm{a}}=10 \mathrm{~T}$ and $\zeta=0.003 \mathrm{~T} / \mathrm{s}$ in adiabatic conditions.

vector $\mathbf{U}$ in the YBCO disk (see Fig. 11). The results were extracted at time $t_{\mathrm{i}}$ corresponding to the beginning of the magnetization, as shown in Fig. 6d, and at the final time $t_{\mathrm{f}}$ when steady-state regime settled. At the beginning of the magnetization, the current density penetrated from the external corners of the bulk to fill up gradually the sample. In steady state regime, the current density had diffused throughout the bulk with a peak value at its outer edge. The temperature was nearly homogeneous during the process due to the slow magnetization rate. The peak of deformation occurred during the magnetization to relax when the background magnetic field vanished. Fig. 6a and $6 \mathrm{~b}$ compare the patterns drawn by the current density at time $t_{\mathrm{i}}$ for both a strain-free and a straindependent $J_{\mathrm{c}}$. Both figures show a sharp diffusion front of the current density although the pattern is distinct. Due to the axisymmetry of the problem, the stress which was obtained from the gradient of mechanical deformation according to 8 tends to increase moving away from the outer stress-free surface. At the axis of symmetry, it reaches its maximum value thereby explaining the loss of the elliptical front of the current density as it diffused to the center of the bulk. This
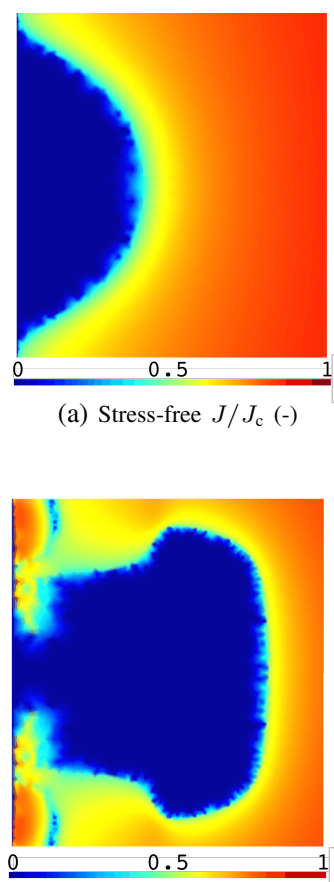

(c) Stress-dependent $J / J_{\mathrm{c}}(-)$, $\vartheta_{\mathrm{F}}=20 \mathrm{MPa}$ (a) Stress-free $J / J_{\mathrm{c}}(-)$

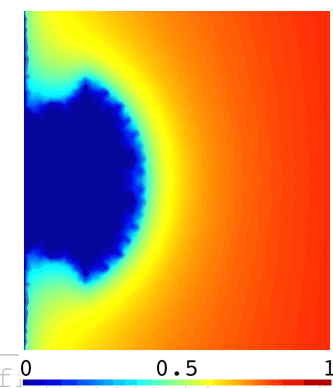

(b) Stress-dependent $J / J_{\mathrm{c}}(-)$, $\vartheta_{\mathrm{F}}=90 \mathrm{MPa}$

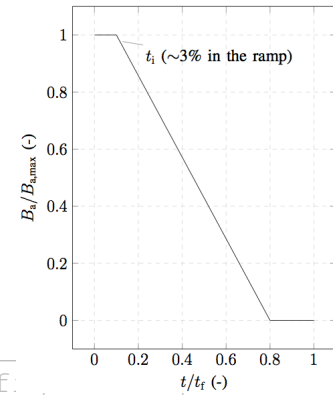

(d) Ramp pattern
Fig. 6. Axisymmetric distribution of $J / J_{\mathrm{c}}$ (-) in the bulk for $T_{\mathrm{op}}=$ $30 \mathrm{~K}, B_{\mathrm{a}}=10 \mathrm{~T}$ and $\zeta=0.003 \mathrm{~T} / \mathrm{s}$ in adiabatic conditions. (a) strain-free $J_{\mathrm{c}}\left(J_{\mathrm{c}, \mathrm{m}}=1\right)$, (b) strain-dependent $J_{\mathrm{c}}$ with $\vartheta_{\mathrm{F}}=90 \mathrm{MPa}$, (c) strain-dependent $J_{\mathrm{c}}$ with $\vartheta_{\mathrm{F}}=20 \mathrm{MPa}$, and (d) ramp pattern of the magnetization.

difference can be clearly appreciated in Fig 6c which shows the current density distribution in the bulk at $t_{\mathrm{i}}$ for a lesser fracture strength equal to $20 \mathrm{MPa}$. For this case, the solution presented convergence issues. However, it is apparent that the front of current diffusion at nearly $J_{\mathrm{c}}$ has been expelled towards the edges of the bulk and the current density in the inner portion has been drastically reduced in comparison to Fig. 6a and 6b

\section{CONCLUSION}

A multiphysics FEM model of YBCO pellets was partly cross-checked with available experimental data. First insights of the impact of the mechanical stress on the distribution of induced current density, at different magnitudes of background magnetic fields and operating temperatures at a given ramp rate, were then obtained. It was shown that the magnitude of the strength fracture should be assessed when it is of the order of the stress generated in the bulk since it affects strongly the distribution of current density. The fracture strength is related to the pre-existence of defects and cracks generated during the growth of the sample and/or during the initial temperature cooling, so it should be estimated at cryogenic temperatures with enough statistics to represent the variability from sample to sample. When the induced stress is of the order of the fracture strength, the numerical model becomes unstable, an issue that should be dealt with to study any limiting cases using the chosen ideal approach. It should be noted that the actual stress-strain curve is expected to show a slight deviation from pure elastic behaviour and the critical current density is likely to degrade at a lower critical strain. 


\section{REFERENCES}

[1] S. Haindl, H.W. Weber, M. Sefciková, K. Zmorayová, P. Diko, N. Hari Babu, D. A. Cardwell, L. Shlyk, G. Krabbes, "Studies of cracking behavior in melt-processed YBCO bulk superconductors," Journal of Physics: Conference Series, vol. 43, pp. 429-433, 2006.

[2] Y. Ren, R. Weinstein, J. Liu, R.P. Sawh, C. Foster, "Damage caused by magnetic pressure at high trapped field in quasi-permanent magnets composed of melt-textured Y-Ba-Cu-O superconductor," Physica $C$, vol. 251, pp. 15-26, 1995.

[3] T.H. Johansen, "Flux-pinning-induced stress and magnetostriction in bulk superconductors," Superconductor Science and Technology, vol. 13, pp. R121-R137, 2000.

[4] F. Trillaud, K. Berger, B. Douine, J. Lévêque, "Comparison between modeling and experimental results of magnetic flux trapped in YBCO bulks," IEEE Transactions on Applied Superconductivity, vol. 26, no. 3, p. $6800305(5 \mathrm{pp}), 2016$.

[5] R. Davidge, Mechanical behaviour of ceramics. Cambridge Solid State Science Series, Cambrige University Press, 1979.

[6] M. Tomita, M. Murakami and K. Yoneda, "Improvements in the mechanical properties of bulk YBCO superconductors with carbon fibre fabrics," Superconductor Science and Technology, vol. 15, pp. 803-807, 2002.

[7] M. Sugano, T. Nakamura, T. Manabe, K. Shikimachi, N. Hirano and S. Nagaya, "The intrinsic strain effect on critical current under a magnetic field parallel to the $c$ axis for a MOCVD-YBCO-coated conductor," Superconductor Science and Technology, vol. 21, p. 115019 (8pp), 2008.

[8] D.C. van der Laan, T.J. Haugan, P.N. Barnes, D. Abraimov, F. Kameani, D.C. Labalestier and M.W. Rupich, "The effect of strain on grains and grain boundaries in $\mathrm{YBa}_{2} \mathrm{Cu}_{3} \mathrm{O}_{7-\delta}$ coated conductors," Superconductor Science and Technology, vol. 23, p. 014004 (7pp), 2010.

[9] T. Anderson, Fracture mechanics - fundamentals and Applications. CRC Press, $3^{\text {rd }}$ ed., 2006.

[10] C. Geuzaine and J.-F. Remacle, "Gmsh: a three-dimensional finite element mesh generator with built-in pre- and post-processing facilities," International Journal for Numerical Methods in Engineering, vol. 79, no. 11 , pp. 1309-1331, 2009.

[11] P. Dular and C. Geuzaine and F. Henrotte and W. Legros, "A general environment for the treatment of discrete problems and its application to the finite element method," IEEE Transactions on Magnetics, vol. 34, no. 5 , pp. $3395-3398,1998$.

[12] F. Inanir, S. Yildiz, K. Oxturk, and S. Celebi, "Magnetization of Gd diffused $\mathrm{YBa}_{2} \mathrm{Cu}_{3} \mathrm{O}_{7-\delta}$ superconductor: Experiment and theory," Chinese Physics B, vol. 22, no. 7, p. 077402 (5pp), 2013.

[13] A. Murakami, H. Teshima, M. Morita, T. Kudo and A. Iwamoto, "Effects of RE compositional boundaries on fracture strength at $77 \mathrm{~K}$ in large single-grained RE-Ba-Cu-O bulk $150 \mathrm{~mm}$ in diameter," Journal of Physics: Conference series, vol. 507, p. 012034 (4pp), 2014.

[14] K. Konstantopoulou, Y.H. Shi, A.R. Dennis, J.H. Durrell, J.Y. Pastor, and D.A. Cardwell, "Mechanical characterization of $\mathrm{GdBCO} / \mathrm{Ag}$ and $\mathrm{YBCO}$ single grains fabricated by top-seeded melt growth at 77 and $300 \mathrm{~K}$," Superconductor Science and Technology, vol. 27, no. 11, p. 115011 (11pp), 2014.

[15] K. Berger, J. Lévêque, D. Netter, B. Douine, and A. Rezzoug, "Influence of Temperature and/or Field Dependences of the $E-J$ Power Law on Trapped Magnetic Field in Bulk YBaCuO," IEEE Transactions on Applied Superconductivity, vol. 17, no. 2, pp. 3028-3031, 2007.

[16] C.Y. Shigue, T.T. da Cruz, J.S. Lamas, C.A. Baldan, and E.R. Filho, "Analysis of the E-J Curve of HTS Tapes Under DC and AC Magnetic Fields at $77 \mathrm{~K}$," IEEE Transactions on Applied Superconductivity, vol. 19 , no. 3, pp. 3332-3335, 2009.

[17] V. Lombardo, E. Barzi, D. Turrioni and A.V. Zlobin, "Critical Currents of $\mathrm{YBa}_{2} \mathrm{Cu}_{3} \mathrm{O}_{7-\delta}$ Tapes and $\mathrm{Bi}_{2} \mathrm{Sr}_{2} \mathrm{CaCu}_{2} \mathrm{O}_{x}$ Wires at Different Temperatures and Magnetic Fields," Fermilab Conference, FERMILABCONF-10-296-TD, pp. 5MX-05 (4pp), 2010.

[18] M. Chudy, Z. Zhong, M. Eisterer and T. Coombs, " $n$-Values of commercial YBCO tapes before and after irradiation by fast neutrons," Superconductor Science and Technology, vol. 28, p. 035008 (10pp), 2015.

[19] T.R. Ravindran, V. Sankaranarayanan, and R. Srinivasan, "Thermal conductivity of $\mathrm{YBa}_{2} \mathrm{Cu}_{3} \mathrm{O}_{7}$," Pramana - Journal of Physics, vol. 39, no. 2, pp. 109-116, 1992.

[20] H. Fujishiro, M. Ikebe, T. Naito, K. Noto, S. Kohayashi and S. Yoshizawa, "Anistropic thermal diffusivity and conductivity of $\mathrm{YBCO}(123)$ and $\mathrm{YBCO}(211)$ mixed crystals. I," Japanese Journal of Applied Physics, vol. 33, no. 9A, pp. 4965-4970, 1994.
[21] R. Jha, P. Rani, V.P.S. Awana, "Revisiting heat capacity of bulk polycrystalline $\mathrm{YBa}_{2} \mathrm{Cu}_{3} \mathrm{O}_{7-\delta}$," Journal of Superconductivity and Novel Magnetism, vol. 27, pp. 287-291, 2014.

[22] M.D. Ainslie and H. Fujishiro, "Modelling of bulk superconductor magnetization," Superconductor Science and Technology, vol. 28, p. 053002 (20pp), 2015.

[23] M. Niffenegger, K. Reichin, "The proper use of thermal expansion coefficients in finite element calculations," Nuclear Engineering and Design, vol. 243, pp. 356-359, 2012.

[24] I. Parinov, Microstructure and Properties of High-Temperature Superconductors. Technology \& Engineering, Springer Science \& Business Media, 2013.

[25] A. Adamowitz, "Axisymmetric FE model to analysis of thermal stresses in a brake disk," Journal of theoretical and applied mechanics, vol. 53, no. 2, pp. 357-370, 2015.

[26] P. Diko, "Growth-related microstructure of melt-grown REBa2Cu3Oy bulk superconductors," Superconductor Science and Technology, vol. 13, no. 3, pp. 1202-1213, 2000. 